\title{
Hexagonal close-packing structure on a cubic cell
}

\author{
N. G. Almarza ${ }^{a)}$ \\ Instituto de Química Física Rocasolano [Consejo Superior de Investigaciones Científicas (CSIC)], \\ C/ Serrano 119, E-28006 Madrid, Spain
}

(Received 23 May 2005; accepted 16 June 2005; published online 9 August 2005)

[DOI: 10.1063/1.1997138]

The solid phases at high pressure of atomistic systems with simple interaction models usually present close-packing structures. These structures are usually described ${ }^{1}$ in terms of stacking of close-packed triangular lattice layers with stacking sequences $\{A B A B A B\}$ for the hexagonal-close-packed (hcp) structure and $\{A B C A B C\}$ for the face-centered cubic (fcc). The relative thermodynamic stability at given conditions of temperature and pressure of these two structures depends on the specific details of the pair potentials. The freeenergy difference between both phases uses to be small and it is often assumed that fcc is the stable crystalline phase; this is due to the fact that the fcc structure can be built up on a cubic cell, which makes the management of periodic boundary conditions (PBCs) easier in simulations, ${ }^{2}$ whereas in the simulation of hcp phases other geometries for the simulation cells are usually chosen. ${ }^{3,4}$ In practice, many simulation codes are restricted to work with cubic boxes. It is therefore interesting to analyze the possibility of building up a hcp structure compatible with a cubic box.

Let us, firstly, remind how the fcc structure can be built in a cubic simulation box of length $L$. We consider systems with $N=4 m^{3}$, with $N$ being the number of sites and $m$ a positive integer number. The particle positions are set to be $\left(k_{x} l, k_{y} l, k_{z} l\right)$, with $l=L /(2 m), k_{x}, k_{y}$, and $k_{z}$ being integers, that fulfill $0 \leqslant k_{\alpha}<2 m$ and $k_{x}+k_{y}+k_{z}$ being an even number. Within this realization of the fcc structure we can consider the different layers introduced above to be perpendicular to the $(1,1,1)$ vector. We can then identify the layer of a given position, $\mathcal{P}(x, y, z \mid m)$, by adding up the site coordinates.

$$
\mathcal{P}(x, y, z \mid m)=(x+y+z) \frac{m}{L} .
$$

When applying Eq. (1) to the site positions of the perfect lattice (as described above) we get integer values of $\mathcal{P}: 0,1,2, \ldots$ In principle, one can apply certain displacements to all the atoms of a number of layers (keeping each atom in its layer plane) to modify the stacking sequence and attain different close-packing structures (e.g., $\{A B C A B C\} \leftrightarrow\{A B A B A B\})$. However, PBCs have to be considered to apply such a strategy correctly. Let $\mathbf{R}^{\prime}$ and $\mathbf{R}$ be the positions of the different images of a given particle:

$$
\mathbf{R}^{\prime}=\mathbf{R}+n_{x} \boldsymbol{L} \mathbf{e}_{x}+n_{y} \boldsymbol{L} \mathbf{e}_{y}+n_{z} \boldsymbol{L} \mathbf{e}_{z},
$$

with $n_{\alpha}$ being integer numbers and $\mathbf{e}_{\alpha}$ a unit vector on the $\alpha$ direction. The layer identifiers $\mathcal{P}$ will fulfill

$$
\mathcal{P}\left(\mathbf{R}^{\prime}\right)=\mathcal{P}(\mathbf{R})+m \sum_{\alpha} n_{\alpha} .
$$

This result implies that the system size (expressed in terms of $m$ ) must be an integer multiple of the periodicity of the stacking modification. In the current case $m$ must be a multiple of 6 .

Therefore, we can use cubic cells with $m=6,12, \ldots$, with fcc arrangement of sites, as starting points to build up hcp lattices; for each lattice site we can compute its layer number [Eq. (1)] and perform, if required for such a layer, the translation of the particle to the corresponding new position in the hcp lattice, and eventually project back into the simulation box the new coordinates.

In practice, it is possible to build up a simple algorithm to perform these operations in a simple way. For a given system size $N=4 m^{3}$ (with $m=6,12, \ldots$ ) we firstly define on the cubic cell of length $L$ a simple cubic lattice with $(6 m)^{3}$ sites located at positions given by $\left(k_{x} \delta l, k_{y} \delta l, k_{z} \delta l\right)$ [with $k_{\alpha}$ $=0,1, \ldots, 6 m-1$, and $\delta l \equiv L /(6 m)]$. A given site will be occupied if it fulfills the following conditions:

$$
\begin{aligned}
& \bmod \left(k_{x}+k_{y}+k_{z}, 6\right)=0, \\
& \bmod \left(\left|k_{\alpha}-k_{\beta}\right|, 3\right)=0 ; \quad \alpha=x, y, z ; \quad \beta=x, y, z, \\
& \bmod \left(\left(k_{x}+k_{y}+k_{z}\right) / 6,3\right)=\bmod \left(k_{\alpha}, 3\right),
\end{aligned}
$$

where all the operations in Eq. (4) are defined over integer numbers.

In conclusion, the algorithm proposed in this work can be useful to perform simple and quick tests on the stability of different close-packing crystal structures of simple solids. Nevertheless, for a rigorous comparison between different phases, one should take into account the possible small departures of the hcp phase from its ideal structure. ${ }^{3}$

\section{ACKNOWLEDGMENTS}

The financial support of the Dirección General de Investigación Científica under Grant No. FIS2004-02954-C03-01 is acknowledged.

\footnotetext{
${ }^{\text {a)} E l e c t r o n i c ~ m a i l: ~ n o e @ i q f r . c s i c . e s ~}$

${ }^{1}$ D. Frenkel and B. Smit, Understanding Molecular Simulation, 2nd ed. (Academic, New York, 2002).

${ }^{2}$ M. P. Allen and D. J. Tildesley, Computer Simulation of Liquids (Clarendon, Oxford, 1987).

${ }^{3}$ S. Pronk and D. Frenkel, Phys. Rev. Lett. 90, 255501 (2003).

${ }^{4}$ D. Frenkel and A. J. C. Ladd, J. Chem. Phys. 81, 3188 (1984); R. J. Speedy, J. Phys.: Condens. Matter 10, 4387 (1998); A. D. Bruce, N. B. Wilding, and G. J. Ackland, Phys. Rev. Lett. 79, 3002 (1997).
} 